\title{
ON THE CONTRIBUTION OF SOLAR ACTIVITY TO THE ULTRAVIOLET SPECTRUM OF THE SUN
}

\author{
A. V. BRUNS, V. K. PROKOFIEV, and A. B. SEVERNY \\ Crimean Astrophysical Observatory, U.S.S.R.
}

\begin{abstract}
As measured from space, the contribution of one moderate flare to the emission spectrum of the Sun in the far-ultraviolet ( $304 \AA$, Lyman continuum, etc.) is comparable with the emission of the whole undisturbed solar disc.
\end{abstract}

The grazing incidence spectrometer of the Crimean astrophysical observatory installed on board Kosmos 166 (flown on June 16, 1967) recorded the solar spectrum in the region 800-950 $\AA$ from June 16, 1967 until July 5, 1967. The spectrometer used a concave grating ( 300 lines $\mathrm{mm}^{-1}$ ) whose radius of curvature was $500 \mathrm{~mm}$, and a scanning system permitting photoelectric recording of the region $800-950 \AA$ (each cycle took $1.5 \mathrm{~min}$ ) with the aid of a copper-beryllium photomultiplier and a suitable countingrate technique. The guiding system kept the solar beam fixed on the slit of the spectrometer with an accuracy of $\pm 10^{\prime}$ (Bruns et al., 1968).

Kosmos 166 also carried X-ray counters installed by Prof. S. L. Mandelstam, who kindly supplied us with observational data of X-ray emissions during the periods of our observations.

About $50 \%$ of all records have been measured and reduced to give the data on the variations of ultraviolet radiation of the Sun. Three types of these variations were found:

(1) Short-term variations connected with solar flares. Figure 1 is an example showing variations connected with a flare of class $1 \mathrm{~N}$, which appeared at $18^{\mathrm{h}} 06^{\mathrm{m}} \mathrm{UT}$, on June 18, 1967 at the Eastern limb (18.06-19.28) (maximum at 18.30). At the same time the X-ray flare was recorded in the corona at a height of about $20000 \mathrm{~km}$ (according to the data of Prof. Mandelstam). We started recording the UV-radiation at 18.45 UT when the intensity was increasing, although the X-ray intensity was decreasing. The maximum of UV radiation was attained at $19^{\mathrm{h}} 10^{\mathrm{m}}$, about $30 \mathrm{~min}$ after the onset of the flare. Such diversity in the run of different line-emissions of flares is probably a quite normal phenomenon (see Hall and Hinteregger, 1968), and it is well known that the yellow coronal line frequently appears around the flare sometimes half an hour after the onset and then remains for a long time.

(2) Moderately slow variations in a matter of a few hours are shown on Figure 2 and are connected with a series of solar flares leading sometimes to intense flares, or to enhanced activity. In the first example (on June 22), the observations covered a period of 9 hours and several brightenings and minor flares were followed by two more intense flares of classes $1 \mathrm{~N}$ and $1 \mathrm{~B}$. The relative increase of intensity in the ultraviolet reaches $25-30 \%$. In the other case (June 17) the recordings covered 6 hours. They began during small flares ( $1 \mathrm{~N}$ and faint brightenings) and ended with two important flares, one of which is a $2 \mathrm{~B}-$ flare. We also had an appreciable increase of intensity (by $15-30 \%$ ) connected with the increase of flare activity. 


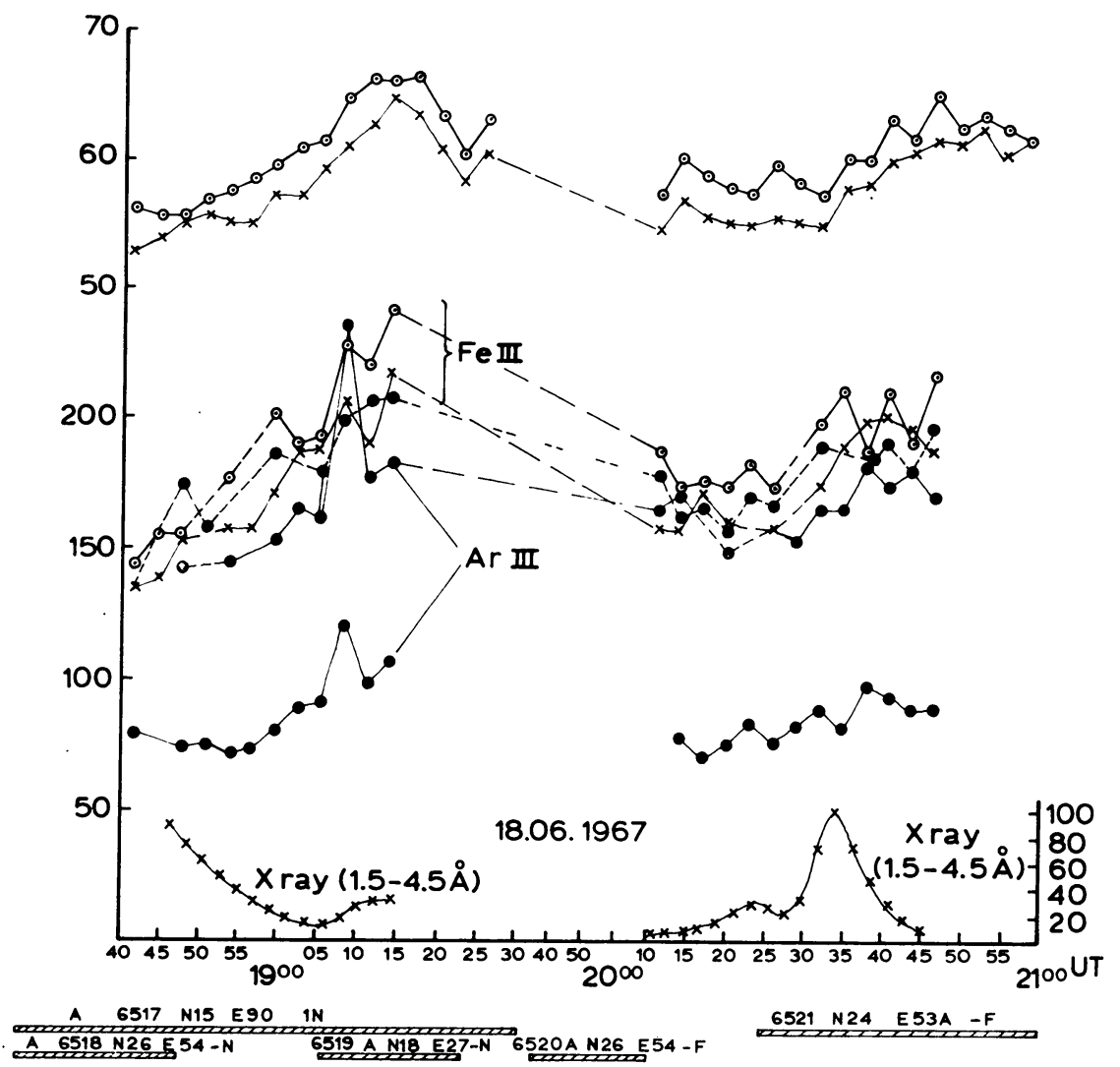

Fig. 1. The intensities (arbitrary units) in the UV regions during the solar flares June 19, 1967. Above: two different parts near the limit of the Lyman series $(920-950 \AA): \times=$ the short wave part, and $\bigcirc=$ the minimum at the middle wavelength. Fe III: three different parts in the region 870-840 $\AA$ : $x=$ the long-wave part, $O=$ the short-wave part, and $\bigcirc=$ at the middle-wavelength. ArIII: the region $875-890 \AA$ : the upper curve $=$ the short wave part, and the lower curve = the long wave part. - Bottom: the durations of solar flares, the moments of their maxima, numbers of group, coordinates of flares and their importance (according to Boulder bulletin) are noted.

(3) Slow day to day variations of ultraviolet radiation of the Sun accompanying the variations in solar active regions. On Figure 3, we show mean values of the intensity (average for each revolution of the satellite around the Earth) for the period from June 16 until July 2, 1967. On the same figure the total area $S_{w}$ of sunspots is also plotted (crosses). There, we have also a relative increase, by $25-40 \%$ the total intensity of the solar disc accompanying the increase of solar activity, provided that the total area of sunspots is adopted as a measure of this activity.

Therefore, we see that solar activity and flares in particular can contribute appreciably (up to $50 \%$ ) to the total flux of ultraviolet emission from the whole disc of the Sun, and it is not impossible that the same kind of activity in stars can produce important changes in the ultraviolet stellar spectra. 


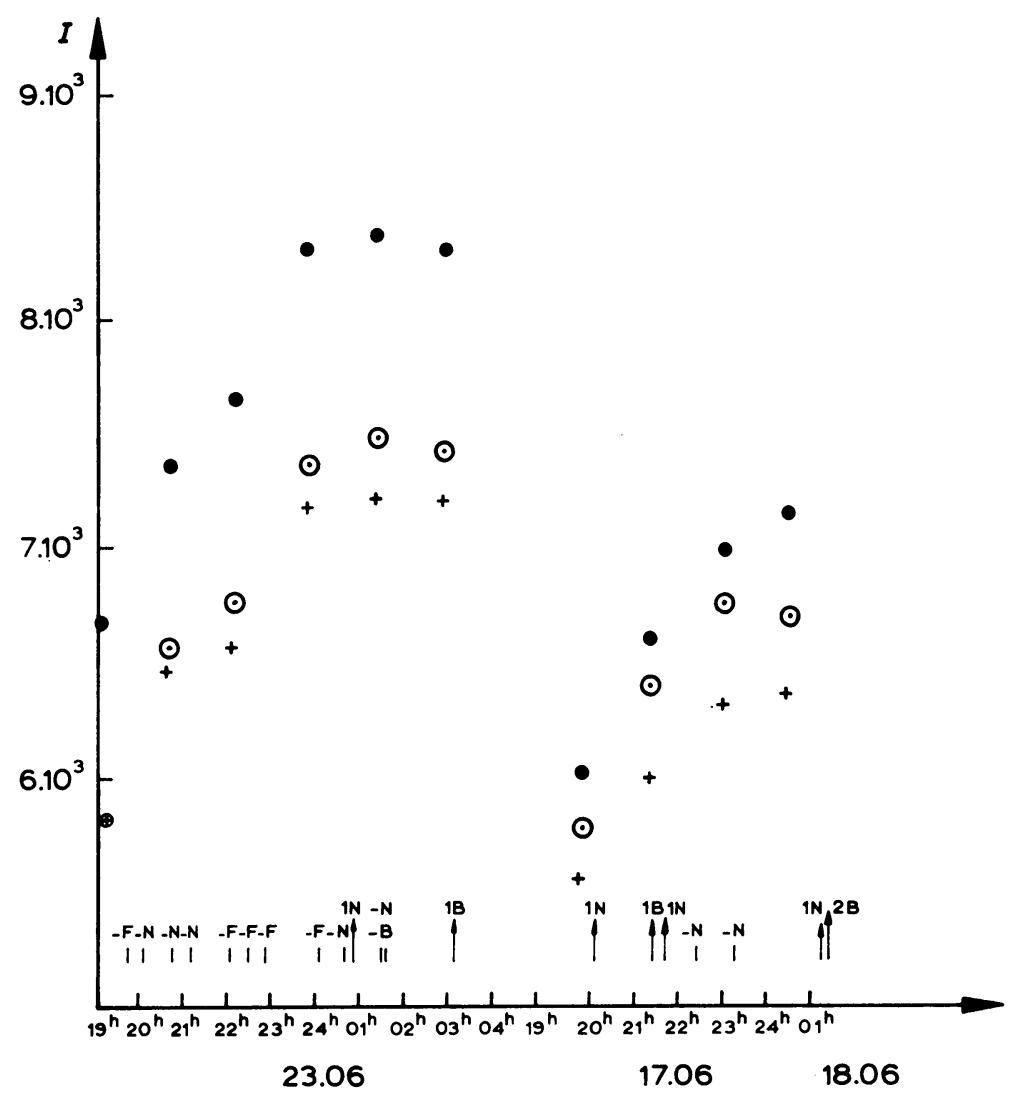

Fig. 2. The intensities in the region of the limit of the Lyman series (920-950 $\AA$ ) during the series of solar flares of June 17 and June 23,1967. $=$ the short wave maximum in this region, $O=$ thelong wave maximum in this region, and $+=$ the minimum of intensity in this region. - Bottom: the moments of onset of solar flares and their importance (from Boulder Bulletin) are noted.

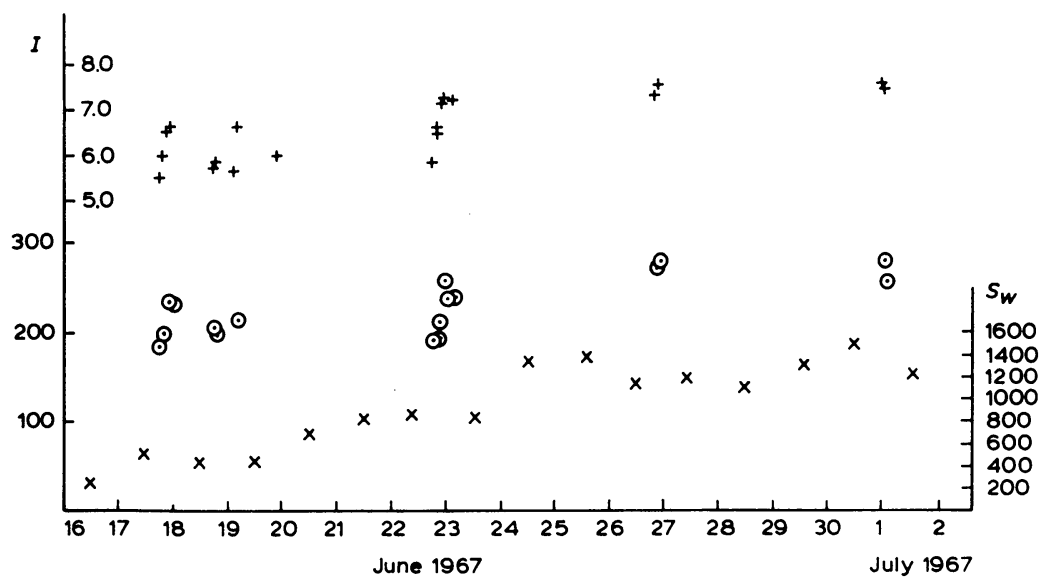

Fig. 3. The intensities in the UV region (arbitrary units) and the area of the sunspots $\left(S_{w}\right)$. $+\ldots$ the region near the limit of the Lyman series $(920-950 \AA) \bigcirc=$ the region of the Fe III lines $(840-870 \AA)$, and the area of the sunspots $\left(S_{u}\right)$ according to Bulletin Solnechnye Dannye. 


\section{References}

Bruns, A. V., Prokofiev, V. K., and Severny, A. B.: 1968, XI COSPAR, Tokyo, May. Hall, L. A. and Hinteregger, H. E.: 1968, XI COSPAR, Tokyo, May.

\section{Discussion}

Jordan: I would like to ask which lines are observed to increase during the flare?

Severny: These lines presumably (not finally identified due to low resolution of our records) are $834 \AA$ (blend of O II, OIII, S III), Fexv $284 \AA$ (from the 3 rd order), $845 \AA, 850 \AA$ Fe III and $878 \AA$ ArIII. Carruthers: Did you see any enhancement of the Lyman continuum in flares?

Severny: Yes, we suspected such enhancements and very preliminary consideration showed that the increase of electron temperature (estimated by the slope of the density curve in the region Lyman $\alpha$ to continuum) can reach $2000-3000 \mathrm{~K}$.

Burton: Is the spectrum observed in the region 800-950 $\AA$ complicated by overlapping spectral lines formed by higher grating orders $(m=3,4)$ ? The high intensity of He II $304 \AA$ and ionized iron emission at about $200 \AA$ is a possible source of blending in the wavelength region which you observe.

Severny: Yes, of course, and we use this blending for rough estimates of intensity changes in $304 \AA$ and $284 \AA$ Fexv. 